\title{
BMJ Open Cost-effectiveness and public health benefit of secondary cardiovascular disease prevention from improved adherence using a polypill in the UK
}

\author{
Virginia Becerra, ${ }^{1}$ Alfredo Gracia, ${ }^{1}$ Kamal Desai, ${ }^{2}$ Seye Abogunrin, ${ }^{2}$ Sarah Brand, ${ }^{3}$ \\ Ruth Chapman, ${ }^{2}$ Fernando García Alonso, ${ }^{1}$ Valentín Fuster, ${ }^{4,5}$ Ginés Sanz ${ }^{5}$
}

To cite: Becerra V, Gracia A Desai $\mathrm{K}$, et al. Costeffectiveness and public health benefit of secondary cardiovascular disease prevention from improved adherence using a polypill in the UK. BMJ Open 2015;5: e007111. doi:10.1136/ bmjopen-2014-007111

- Prepublication history and additional material is available. To view please visit the journal (http://dx.doi.org/ 10.1136/bmjopen-2014007111).

Received 4 November 2014 Revised 16 March 2015 Accepted 13 April 2015

CrossMark

For numbered affiliations see end of article.

Correspondence to

Ginés Sanz;

ginessanzr@gmail.com

\section{ABSTRACT}

Objective: To evaluate the public health and economic benefits of adherence to a fixed-dose combination polypill for the secondary prevention of cardiovascular (CV) events in adults with a history of myocardial infarction (MI) in the UK.

Design: Markov-model-based cost-effectiveness analysis, informed by systematic reviews, which identified efficacy, utilities and adherence data inputs.

Setting: General practice in the UK.

Participants: Patients with a mean age of 64.7 years, most of whom are men with a recent or non-recent diagnosis of $\mathrm{Ml}$ and for whom secondary preventive medication is indicated and well tolerated.

Intervention: Fixed-dose combination polypill (100 mg aspirin, $20 \mathrm{mg}$ atorvastatin and 2.5, 5, or $10 \mathrm{mg}$ ramipril) compared with multiple monotherapy.

Primary and secondary outcome measures: $\mathrm{CV}$ events prevented per 1000 patients; cost per life-year gained; and cost per quality-adjusted life-year (QALY) gained.

Results: The model estimates that for each $10 \%$ increase in adherence, an additional $6.7 \%$ fatal and non-fatal CV events can be prevented. In the base case, over 10 years, the polypill would improve adherence by $\sim 20 \%$ and thereby prevent 47 of $323(15 \%)$ fatal and non-fatal CV events per 1000 patients compared with multiple monotherapy, with an incremental costeffectiveness ratio (ICER) of $£ 8200$ per QALY gained. Probabilistic sensitivity analyses for the base-case assumptions showed an $81.5 \%$ chance of the polypill being cost-effective at a willingness-to-pay threshold of $£ 20000$ per QALY gained compared with multiple monotherapy. In scenario analyses that varied structural assumptions, ICERs ranged between cost saving and £21 430 per QALY gained.

Conclusions: Assuming that some 450000 adults are at risk of $\mathrm{MI}$, a 10 percentage point uptake of the polypill could prevent $3260 \mathrm{CV}$ events and $590 \mathrm{CV}$ deaths over a decade. The polypill appears to be a costeffective strategy to prevent fatal and non-fatal CV events in the UK.

\section{Strengths and limitations of this study}

- A polypill that reduces dosing complexity has been proposed as a way of improving adherence and outcomes in patients with existing cardiovascular (CV) disease, but clinical evidence from long-term trials confirming such benefit has not as yet been published. Also, the economic acceptability and the public health potential of such a polypill have not as yet been evaluated for the UK.

- The modelling approach employed here suggests in the base case that use of a fixed-dose combination polypill that improves adherence by $20 \%$ over multiple monotherapy in patients with myocardial infarction (MI) could prevent 47.3 fatal and non-fatal CV events per 1000 population over a 10-year period and is likely to be cost-effective at acceptable willingness-to-pay levels in the UK.

- For each $10 \%$ improvement in the proportion of adherent patients, an additional $6.7 \%$ of fatal and non-fatal CV events could be prevented. Also, assuming a $10 \%$ uptake of the polypill that improves adherence by $20 \%$ among patients with $\mathrm{MI}$ in the UK, $3260 \mathrm{CV}$ events and $590 \mathrm{CV}$ deaths could be prevented over a decade.

- Models are reductionist in nature and simplify the actual clinical decision-making and biological mechanisms of treatment. In particular, the study does not consider either individualisation of therapeutic dosing adjustments or treatment switching due to adverse events. Therefore, results should be interpreted in the context of patients for whom the polypill is suitable as a replacement for multiple monotherapy and well tolerated, and in the absence of other preventive interventions.

\section{INTRODUCTION}

Cardiovascular (CV) disease remains a major cause of morbidity and mortality in the UK. Between $9 \%$ and $11 \%$ of adults in the UK have existing CV disease, and more than 4 million people are at high risk of having $\mathrm{CV}$ 
events. ${ }^{12}$ The trend of increasing age and obesity in the UK is likely to exacerbate the problem over the next 20 years. ${ }^{3}$ The acknowledged efficacy of secondary preventive medication is reflected in clinical guidelines from the National Institute for Health and Care Excellence (NICE), which recommend that all patients who have had a myocardial infarction (MI) should be offered an ACEI, dual antiplatelet therapy, a statin and a $\beta$-blocker. ${ }^{4}$ Patients who consistently take this combination therapy have significantly reduced risks of subsequent MI, strokes and transient ischaemic attacks (TIAs) and death, compared with those who take only one or two of these drugs. ${ }^{5}$

Although the use of effective secondary preventive medication is increasing, there is still a substantial unmet need in the UK population. For instance, fewer than two-thirds of patients with coronary heart disease (CHD), and a third of those with a stroke or TIA were receiving triple preventive therapy in $2005,{ }^{67}$ and hypertension and hypercholesterolaemia remain inadequately controlled. ${ }^{8}{ }^{9}$ Not only is prescribing inadequate, but adherence to secondary preventive therapy is also low and declines over time. ${ }^{10} 11$ The clinical impact of this poor adherence is substantial. A statistically significant increase in the risk of all-cause mortality, recurrent MI, stroke or TIA and readmissions for recurrent CV events has been demonstrated for those with poor adherence compared with those who take more than $80 \%$ of the prescribed medication. ${ }^{10-15}$ Effective strategies to improve clinical outcomes should be evaluated in a longterm horizon and, where cost-effectiveness is demonstrated, adopted without further delay.

Adherence to medication is affected by various factors including the complexity of the treatment regimen, socioeconomic status, lifestyle and psychological influences. ${ }^{16}$ With regard to the factors that can be modified to improve adherence, the European Society of Cardiology states that 'reducing dosage demands is the most effective single approach'. ${ }^{17}$ Reducing dosing complexity can be achieved by the use of a fixed-dose combination polypill that contains the recommended treatments in a single daily capsule. This approach has been suggested as a way of improving adherence and reducing costs to the patient. ${ }^{18-22}$ For this purpose, various polypills have been suggested, from the combination of aspirin, a thiazide, a $\beta$-blocker, an ACEI, a statin and folic acid, originally suggested by Wald and Law in 2003 for primary prevention, to a simpler and more evidencebased formulation containing an aspirin, a statin and an ACEI for the secondary prevention of CV events, which was developed in Spain by a private-public partnership between the Centro Nacional de Investigaciones Cardiovasculares (CNIC) and Ferrer Internacional. ${ }^{20}$ This latter polypill includes $100 \mathrm{mg}$ aspirin, $20 \mathrm{mg}$ atorvastatin, and $2.5,5$ or $10 \mathrm{mg}$ ramipril.

To date, no findings from large randomised controlled trials (RCTs) comparing the efficacy of a polypill with multiple monotherapy in reducing long-term GV events have been published. We have therefore developed a model, informed by a series of systematic literature reviews, to explore the likely public health and economic benefits of the polypill of $100 \mathrm{mg}$ aspirin, $20 \mathrm{mg}$ atorvastatin, and $2.5,5$ or $10 \mathrm{mg}$ ramipril, prescribed in a general practice setting for the secondary prevention of CV events in adults in the UK with a history of MI, compared with its individual components given concomitantly at equivalent doses. The model predicts the number of secondary CV events that are preventable, as well as the incremental costs and benefits in terms of quality of life in a cohort of patients taking the polypill.

\section{METHODS}

\section{Economic model design}

A Markov model with a 3-month cycle length was developed in Microsoft Excel (2007) to predict the CV outcomes, costs and benefits to estimate the incremental cost-effectiveness ratios (ICERs) per life-year (LY) gained and per quality-adjusted life year (QALYs) gained from the start of treatment until the analytic time horizon (10 years) or death. The analysis adopted the perspective of the National Health Service (NHS) and the Personal Social Services of the UK. ${ }^{23 a}$ The population included in the model was intended to match closely the characteristics of patients in whom secondary prevention with aspirin, atorvastatin and ramipril is indicated and well tolerated. The population had a mean age of 64.7 years and was $72 \%$ male, with a recent or non-recent diagnosis of acute MI. ${ }^{22}$ The model allowed the patients to have one of five $\mathrm{CV}$ events: non-fatal acute coronary syndrome events (ACS, combining recurrent MI or unstable angina); non-fatal stroke; non-fatal congestive heart failure (CHF) requiring hospitalisation; unplanned revascularisation procedures; or CV death. In addition, patients could experience non-CV-attributable death. The model diagram is given in figure 1 . Key model equations determined the rates at which CV events occur as a function of baseline risks, adherence to the polypill, aspirin, statin and ACEI and relative risk reductions (RRR) of each of the three medications specific to each CV event type for adherent and nonadherent patients (see online supplementary appendix 1 for detailed assumptions, additional model description and equations). The five CV event outcomes were undiscounted to allow for a more natural interpretation of the result in the public health context, while outcomes for costs, LYs, QALYs and ICERs were discounted in the base case at $3.5 \%$, in line with the NICE reference case. $^{23}$

\section{Input data}

All data required to inform the model structure and parameters were based on the findings of a series of systematic reviews of studies reporting on the efficacy of aspirin, ACEI and statins as secondary prevention in 


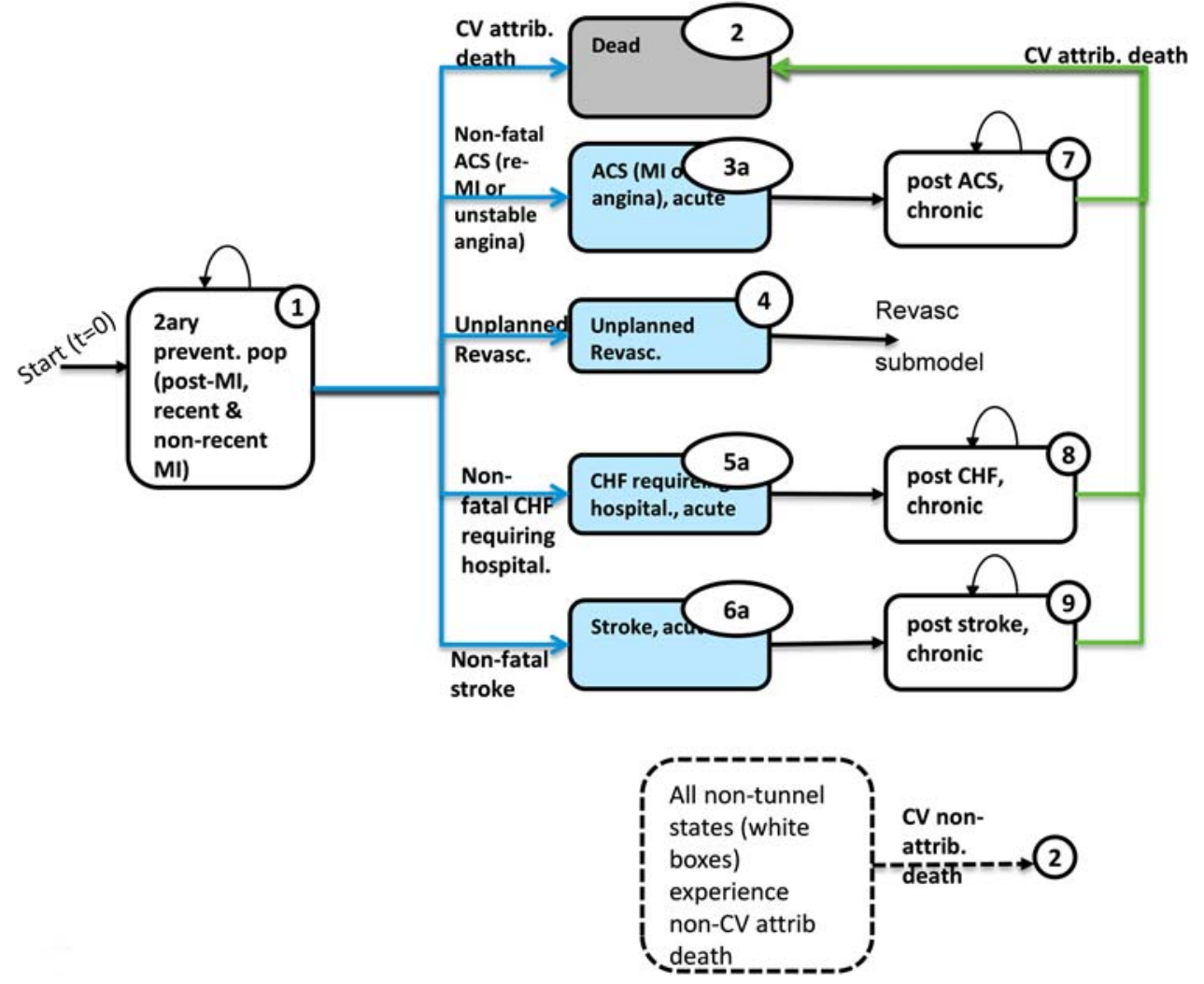

Figure 1 Markov model of secondary prevention of cardiovascular events in patients surviving a first myocardial infarction. ACS, acute coronary syndrome; CHF, congestive heart failure; CV, cardiovascular; ICER, incremental cost-effectiveness ratio; MI, myocardial infarction.

patients with existing CV disease; the costs, resource use and utility values associated with existing CV disease, and secondary prevention; adherence to secondary prevention regimens; and existing economic models of secondary prevention therapies. We ran systematic searches in PUBMED, EMBASE, the NHS Economic Evaluation Database (NHSEED) and the Cochrane Library for relevant studies published in English between January 2003 and July 2013, and screened these using predefined inclusion criteria. (Online supplementary appendices 2 and 3 describe the search strategy and inclusion/exclusion criteria for the literature review, respectively.) This identified 1835 unique publications for the efficacy review and 4418 unique publications for the other topics. Of these, 56 papers were retrieved for the efficacy review, of which 29 were included in the review, and 287 papers were retrieved for the other topics, of which 192 were included in the review. (Online supplementary appendix 4 shows the Preferred Reporting Items for Systematic Reviews and Meta-Analyses (PRISMA) diagram of the flow of literature in the review.) We also made a call for evidence within Ferrer and CNIC, which identified an additional 10 publications. Abstracts of efficacy studies were screened independently by two researchers and differences in screening decisions were reconciled by discussion with a third researcher. For the other topics, potentially relevant primary studies were identified by one researcher. Full-text versions of all shortlisted studies were checked for relevance by two researchers, with guidance from medical experts.

\section{Efficacy}

The systematic approach permitted estimation of the efficacy of aspirin, statins and ACEI for the five clinical outcomes to be derived from existing meta-analyses. This confirmed the reduction in CV events with ACEI, aspirin and statins, with relative risk reductions generally between 0.6 and 0.8 . No study in our search had evaluated clinical outcomes associated with a polypill, and we therefore assumed that the relative risk reductions were similar for multiple monotherapy and a polypill. (See online supplementary appendix 1: table 1 for details of further assumptions used in the model).

\section{Adherence}

Most studies reporting adherence used a cut-off of taking $80 \%$ or more of prescribed doses in their definition of good adherence, thereby dichotomising patients as adherent or not. Overall adherence to multiple monotherapy regimens as a free combination was approximately 50-60\% after the first year, and poor adherence to any or all the monocomponent drugs was associated with worse clinical outcomes. There was considerable variability across studies in adherence rates and population characteristics, so base-case inputs for the model on drug adherence were based on the 
UMPIRE study, ${ }^{24}$ in which $86 \%$ of patients were adherent to the polypill compared with $65 \%$ who were adherent to all three drugs as multiple monotherapy at a median follow-up of 15 months. The adherent proportion was modelled according to an initial maximum value, which declined over 15 months to reach a fixed, constant value thereafter. Importantly, adherence in the monocomponents arm followed the same basic equation as the polypill arm but with different parameter values. Hence, all patients were assumed to be adherent to all three drugs. Adherence assumptions were explored in extensive sensitivity analyses.

\section{Costs, event rates and utilities}

In line with the chosen perspective, costs and management of CV events were obtained from NHS reference costs. The British National Formulary (BNF) provided the costs of the drugs. The cost year was 2014. CV event rates, utilities and other costs were informed by selected published clinical trials, previous economic models or registry studies. Base-case clinical and economic parameter values and their sources are shown in table 1 .

\section{Model assumptions}

All structural and data assumptions that were made are presented in online supplementary appendix 1. Two observers reviewed the structure, assumptions of the model and data sources that informed the model parameters. The main structural assumptions included the following: the model risk equations for $\mathrm{CV}$ events assumed that baseline risks and efficacy among adherent patients were equal for the polypill and its monocomponents in terms of health benefits, and that differences in outcomes are generated exclusively by differences in adherence; patients were assumed to be either adherent or not; and the benefit of medication was multiplicative (ie, RRRs of individual drugs were multiplied together for adherent patients). Also, the model does not take account of adverse effects of medication or individualisation of therapy that allows for dose adjustments or medication switches, because it assumes that the patients being prescribed specific monocomponents or a polypill are receiving a suitable and tolerated secondary preventative therapy.

\section{Base-case and sensitivity analyses}

Public health outcomes presented as CV events prevented per 1000 patients were determined for the base-case. In addition, the total percentage of CV outcomes preventable per 1000 patients was calculated as a function of the different relative improvements in adherence with the polypill compared to the monocomponents. The base-case analysis also compared costs and QALYs to determine the incremental cost per QALY gained (table 2). Given the uncertainty from the literature on the persistence of medication adherence, 10 years was chosen as the base-case time horizon, to correspond with a plausible duration of

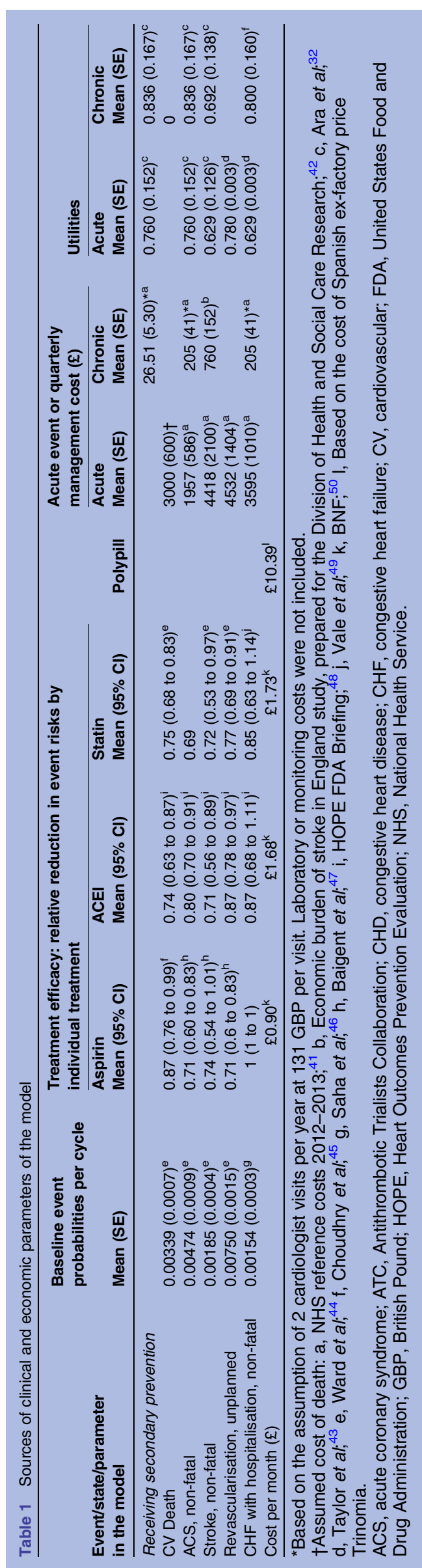


Table 2 Undiscounted public health and discounted economic outcomes in base-case analysis (per 1000 population)

\begin{tabular}{llll}
\hline CV disease events and economic outcomes & Polypill & Monocomponents & $\begin{array}{l}\text { Difference } \\
\text { (\% reduction/gain) }\end{array}$ \\
\hline ACS events & 61.06 & 75.31 & $-14.25(-21.9)$ \\
Revascularisation (unplanned and unrelated to other CV events) & 104.49 & 120.76 & $-16.26(-15.5)$ \\
Congestive heart failure with hospitalisation & 32.35 & 33.86 & $-1.51(-5.2)$ \\
Stroke & 23.20 & 28.90 & $-5.70(-22.8)$ \\
CV death & 54.62 & 64.19 & $-9.57(-17.3)$ \\
Total LY (discounted) & 6338.57 & 6307.69 & $30.88(0.5)$ \\
Total QALY (discounted) & 5278.46 & 5248.92 & $29.54(0.6)$ \\
Drug costs (discounted) & $£ 790229$ & $£ 326701$ & $£ 463528(141)$ \\
Cost of acute CV events and deaths (discounted) & $£ 2064865$ & $£ 2195567$ & $-£ 130702(-6.0)$ \\
Cost of patient management (discounted) & $£ 1139719$ & $£ 1230203$ & $-£ 90484(-7.4)$ \\
Total costs (discounted) & $£ 3994814$ & $£ 3752473$ & $£ 242341$ \\
ICER (discounted) & - & - & $£ 8205$ per QALY \\
\hline ACS, als
\end{tabular}

ACS, acute coronary syndrome; CV, cardiovascular; ICER, incremental cost-effectiveness ratio; LY, life-years; QALYs, quality-adjusted life-years.

improved adherence. To identify model drivers and examine key areas of uncertainty within the model, one-way deterministic sensitivity analyses were conducted for all major model variables. To account for multivariate and stochastic uncertainty in the model, a probabilistic sensitivity analysis (PSA) was performed with 10000 simulations.

Multiple scenario analyses were performed to study the effects of the following variations in structural and cost assumptions:

- Patients in the monocomponents arm may be adherent to three, two, one or no drugs, unlike the polypill arm where patients are adherent to three or no drugs;

- The time horizon for the model is a lifetime, recognising the lifelong nature of CV disease and management costs;

- Patients in the monocomponents arm who are not adherent do not incur costs of unused medication;

- Adherence continues to decline indefinitely at the same rate as observed during the first 15 months, in the polypill and monocomponent arms;

- Polypill adherence is assumed to wane until equalling the long-term adherence of the monocomponent arm which is constant from 15 months;

- Adherence to the polypill exceeds that to monocomponents by between 15 and 25 percentage points;

- As an alternative to UMPIRE, adherence data from Kanyini GAP $^{25}$ (reporting $73.8 \%$ adherence in the polypill arm compared with $49.1 \%$ taking monocomponents at 12 months) are used;

- The unit cost of polypill is varied, first between $£ 9$ and $£ 12$ per month, and second, the cost of the polypill is changed to be equal to ( $£ 4.32$ per month) or double (£8.63 per month) the cost of the individual components.

This economic evaluation was conducted in accordance with the Consolidated Health Economic Evaluation Reporting Standards (CHEERS) Statement. ${ }^{26}$

\section{RESULTS}

Public health outcomes

It is predicted in the base case that a total of 323 and 276 fatal and non-fatal CV events will occur per 1000 patients in the monocomponents and polypill arms, respectively, such that a total of $47.3(14.6 \%)$ CV events (comprising 14.2 ACS events, 16.3 unplanned revascularisations, 1.5 congestive heart failures requiring hospitalisation, 5.7 strokes and 9.6 CV deaths) can be prevented over a period of 10 years by using the polypill in place of its monocomponents taken as multiple monotherapy. The absolute number of CV events in, and the difference between, the intervention groups are given in table 2.

More generally, each $10 \%$ absolute increase in adherence over the monocomponents (eg, an increase 65\% to $75 \%$ ) would prevent an additional $6.7 \%$ of $\mathrm{CV}$ events in 10 years, as shown in figure 2.

\section{Economic outcomes}

\section{Base case}

The total discounted incremental LYs gained in the polypill arm compared to monocomponents per 1000

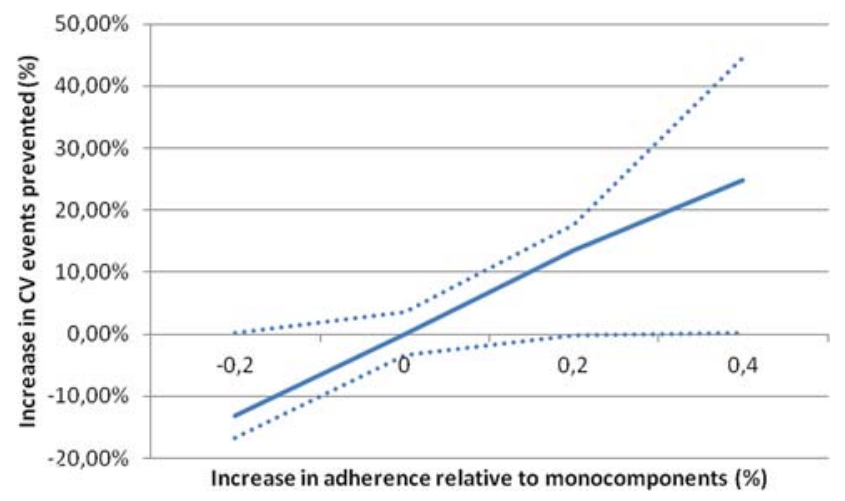

Figure 2 Percentage decrease in total cardiovascular events with the polypill relative to monocomponents. 
patient populations over the 10-year time horizon was 30.88 , and the total incremental QALYs gained were found to be 29.54. The total discounted costs for the polypill were $£ 3994800$ compared with $£ 3752500$ in the monocomponents arm. The corresponding incremental cost of $£ 242300$ consisted of $£ 463500$ in drug costs that were offset by savings of $£ 130700$ and $£ 90500$ due to reduced CV events and patient management. The ICER was $£ 8200$ per QALY gained.

\section{Sensitivity analyses}

One-way sensitivity analyses showed incremental costs to be most sensitive to polypill adherence, discount rate and revascularisation costs, while incremental QALYs and ICERs were most sensitive to utility values of patients on secondary prevention and patients having had a second MI. Probabilistic analyses for the base-case assumptions showed that the chances of the polypill being costeffective were $81.5 \%$ and $84.8 \%$ at willingness-to-pay thresholds of $£ 20000$ and $£ 30000$ per QALY gained, respectively. Further results for the probabilistic sensitivity analysis, including tornado and scatter plots, are given in online supplementary appendix 5. Under alternative scenarios, ICERs were $£ 21400$ when adherence to none, one, two or three of the drugs was allowed in the monocomponents arm; $£ 5300$ when the time horizon was a lifetime; $£ 8300$ when non-adherent patients did not incur full medication costs; $£ 1000$ when adherence in both arms declined indefinitely; $£ 15200$ when polypill adherence waned after 15 months until equalling monocomponent adherence; and $£ 6000$ when assuming adherence results from the Kanyini GAP study (table 3). When varying the long-term adherence of the polypill to between 15 and 25 percentage points higher than with the monocomponents, the ICER ranged between $£ 15100$ and $£ 5900$ per QALY. When the polypill unit cost ranged between $£ 9$ and $£ 12$ per month supply, the ICER ranged between $£ 4900$ and $£ 12700$ per QALY (see online supplementary appendices for figures). The ICER was $£ 3700$ when the polypill price was double that of the monocomponents. The polypill was the dominant strategy when its price was equal to that of the monocomponents, providing greater benefits at a lower overall cost.

\section{DISCUSSION}

We developed an economic model to investigate the public health benefit and cost-effectiveness that could be expected from the improved adherence achieved with a three-in-one polypill compared with multiple monotherapy for secondary prevention of cardiovascular disease. Based on inputs derived from systematic literature reviews, the model has shown that $47 \mathrm{CV}$ events and $10 \mathrm{CV}$ deaths could be prevented in the UK with the polypill compared with multiple monotherapy per 1000 patients over 10 years, at an ICER of $£ 8200$ per QALY gained. In the context of persistent inadequate prescribing despite robust evidence-based guidelines, and poor

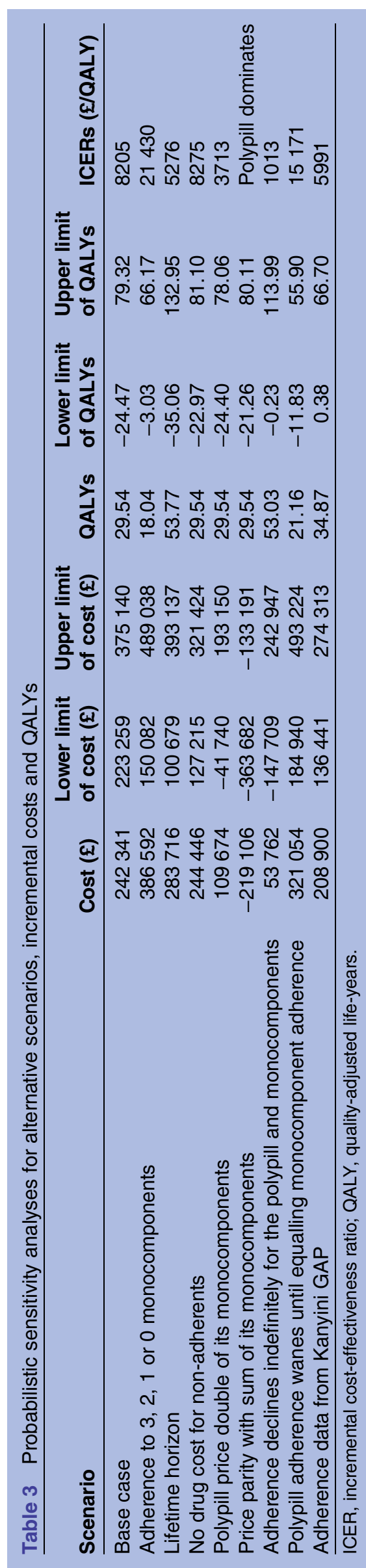


adherence in those receiving multiple monotherapy, our data suggest that the polypill offers a cost-effective approach to improving outcomes for a substantial proportion of the UK population.

In considering our results, it is important to note that multiple patient-related factors affect adherence to medication, and while many of these (such as age, gender, ethnicity and previous medication compliance history) cannot be changed, others, such as disease knowledge, social support and tobacco and alcohol consumption, can be altered over time. Crucially, there is also evidence that medication complexity has an adverse effect on adherence in chronic disease, ${ }^{27} 28$ such that products requiring a simpler regimen are likely to be associated with greater patient adherence than are more complicated regimens. Moreover, it is plausible (although unproven) that the simplified prescribing offered by a polypill could also help to improve current suboptimal adherence to prescribing guidelines among healthcare providers, which may occur, for example, due to physicians' beliefs that dosing schedules associated with multiple monotherapy are overcomplicated.

There are, however, various limitations in simplifying such a complex clinical pathway, including data uncertainties, within a model as we have done. These include the assumption that adherence rates for each of the monotherapy components will be consistently either good or poor in any one individual and that there is no clinical benefit with individual medication adherence rates below $80 \%$. In practice, adherence may vary by drug in any one individual and there is likely to be a continuous reduction in efficacy with falling adherence. However, the cost-effectiveness of the polypill was £21 400 per QALY in sensitivity analyses that allowed a proportion of patients in the monocomponents arm to be adherent to just one or two monocomponents. We took baseline $\mathrm{CV}$ disease risk from that reported in clinical trials, which may underestimate true CV event rates, but this assumption is conservative since higher rates would make the polypill more cost-effective. We assumed relative-risk reductions to be constant over time and, in the absence of data, we assumed no benefit for aspirin in reducing CHF with hospitalisation. It is important to note, however, that the inputs and effect sizes used in our model compare well with those used previously by Gaziano et al..$^{29}$ We also made assumptions about resource use for monitoring patients receiving secondary prevention, which were supported by recommendations from NICE and the Quality and Outcomes Framework, as well as expert opinion. ${ }^{4}$ Of significance, the polypill modelled prices for the combination therapy of aspirin, atorvastatin and ramipril, which are widely used in the UK for secondary prevention, although simvastatin is a more widely prescribed statin overall. ${ }^{30}$ As atorvastatin becomes more widely available as a generic drug in the UK, any cost differences and related variation in cost-effectiveness from the use of different statins will be minimised. Importantly, we used adherence data from the UMPIRE trial, ${ }^{24}$ which assessed a four-in-one polypill, rather than the three-in-one polypill used in our model. Although adherence to the polypill should be similar, adherence to four monocomponents may be lower than adherence to three, so our model may have overestimated the difference in adherence in the two groups. However, we assumed that all patients would also be taking $\beta$-blockers and second antiplatelets as appropriate, so the effects of these were assumed to cancel out when the two groups were compared. This exclusion may have had only a slight impact on our conclusions, given that inclusion of a $\beta$-blocker monocomponent taken concomitantly with the polypill or the other three monocomponents with the same adherence in both arms will not produce additional cost differences and may produce only marginal advantages in effectiveness compared with a tripletherapy regimen. The EURO-ASPIRE III study found that patients with a previous MI, seen in routine clinical practice, do not achieve targets as set by established guidelines. ${ }^{31}$ These include therapeutic, lifestyle and risk factors. Our model, however, only captured the benefits associated with therapeutic compliance and adherence because we assumed that effects on lifestyle changes would be similar in both treatment groups. Accordingly, we did not estimate the incremental effects of attaining additional modifiable lifestyle and risk factor goals in the patients we evaluated. We also did not consider individualisation of therapeutic dosing adjustments or treatment switching due to adverse events. The results of the analysis should, therefore, be interpreted in the context of patients for whom the polypill is suitable as a replacement therapy versus the monocomponents and well tolerated, in the absence of other preventive interventions.

Although a number of other economic models for secondary prevention have been developed, many of these assessed effectiveness of individual drugs or combinations of drugs compared with no therapy or with different doses or therapeutic combinations, implying different clinical efficacies between interventions. ${ }^{29}{ }^{32-36}$ Our analysis differs from others in assuming that the therapies are identical in terms of clinical efficacy among adherent patients, but allowing adherence rates to determine differences in CV events and economic outcomes. Our model was consistent with previous ones in terms of time horizon, which ranged from 2 years to a lifetime, with 7 of the 15 models using a lifetime horizon and another 4 using a 10-year horizon. However, we also conducted a lifetime analysis in a sensitivity analysis to reflect the lifelong nature of CV disease and treatment. Our model structure was generally consistent with other models since health states usually included stable disease, recurrent CV events and death. Under the base-case and sensitivity analyses, which varied costs of treatment, the efficacy of interventions, adherence, utility and population characteristics, secondary prevention with the polypill in adult patients with a recent or non-recent diagnosis of MI and eligible for secondary prevention with aspirin, statins and ACEI is a cost-effective strategy at 
acceptable willingness-to-pay thresholds in the UK, given that ICERs were below or near the $£ 20000$ per QALY threshold.

The public health benefit suggested by our model depends on an assumed marked increase in adherence to secondary prevention therapy through use of the polypill, based on the UMPIRE study, which demonstrated that a $20 \%$ increase in adherence is possible. Our assumption about increased adherence is also in line with the very recent results from the IMPACT trial, part of the 'Single Pill Against Cardiovascular Events (SPACE)' collaboration, which demonstrated a significant difference in adherence in patients taking a four-in-one polypill (81\%) versus the usual care group (46\%), at 12 months. ${ }^{37}$

Our model estimates that for each $10 \%$ increase in adherence, an additional $6.7 \%$ fatal and non-fatal CV events can be prevented. The potential of this result for the eligible UK population is striking: specifically, 3260 CV events and 590 deaths could be prevented in the next decade. This is based on a modified version of our model that assumes that the prevalence of MI survivors aged 25 years and older is $1.4 \%$ (as inferred from the British Heart Foundation data) $;{ }^{38}$ the incidence of MI is $0.112 \%$ (as derived from Smolina) ${ }^{39}$ there are 45271000 in this age group among the UK population; ${ }^{40}$ and that $10 \%$ of eligible patients switch from monotherapy to the polypill and achieve a $20 \%$ improvement in adherence. If uptake of the polypill is more than $10 \%$ in those eligible or patients at high risk of a first MI also receive the polypill, the public health benefit would be even greater.

In summary, our evaluation provides key evidence on an important issue in clinical management: the fact that, while the efficacy of secondary prevention in CV disease is well established in patients adherent to medication, adherence is generally low, and this has a negative impact on clinical outcomes. Despite its analytic limitations, our study suggests that a three-in-one polypill that improves adherence and outcomes in patients with existing CV disease would have a positive public health impact in the UK especially in the context of inadequacies in physician prescribing and patient adherence. It could therefore become the mainstay of secondary cardiovascular prevention for patients in whom such triple therapy is suitable. Future research is needed from long-term RCTs to confirm whether this approach offers advantages over multiple monotherapy in preventing $\mathrm{CV}$ events in patients who have experienced an MI.

\section{Author affiliations}

${ }^{1}$ Ferrer, Barcelona, Spain

2Evidera, Hammersmith, London, UK

${ }^{3}$ Evidera, Bethesda, Maryland, USA

${ }^{4}$ The Mount Sinai Medical Center, New York, New York, USA

${ }^{5}$ Centro Nacional de Investigaciones Cardiovasculares (CNIC), Madrid, Spain

Acknowledgements The authors would like to acknowledge the assistance of Jaume Marrugat and Roberto Elosua for the review of structure, assumptions and data sources to inform the model parameterisation; Juan Carlos Kaski for his expert advice regarding current UK clinical practice in the management of
CV disease; and Alison Martin, Liliana Falkon and Lisette Kaskens for their editorial support and assistance at various stages of the systematic review.

Contributors VB and AG participated in the design of the systematic literature review, model conceptualisation and specification, planned the model analyses and critically reviewed the search strategy for the systematic review and appraised articles for inclusion. FG-A participated in the model conceptualisation, critically reviewed the search strategy and appraised articles for inclusion. GS participated in the design of the systematic literature review, model conceptualisation and specification, critically reviewed the search strategy for the systematic review and appraised articles for inclusion. He also analysed the clinical data finding the model and helped in the interpretation of results from the clinical point of view. KD and $\mathrm{RC}$ developed the model conceptualisation, performed the model specification and calculated the algorithms for model implementation. SB programmed the economic model, and performed and recorded model analyses, including base case results and probabilistic and deterministic sensitivity analyses. SA designed and conducted the search for the systematic review, screened and appraised articles for inclusion, abstracted data and synthesised the findings. VF reviewed the results from the clinical point of view. VB, AG, GS and KD devised and drafted the manuscript. All authors had full access to all the data in the study; all authors were responsible for the decision to submit for publication. All authors reviewed the final draft of the manuscript.

Funding This work was supported by a full grant from Ferrer Internacional.

Competing interests $\mathrm{KD}, \mathrm{SA}, \mathrm{SB}$ and $\mathrm{RC}$ are employees of Evidera, a consultancy hired by Ferrer Internacional to perform the systematic literature review (SLR) and development of the model. VF and GS are members of CNIC where the fixed-dose combination polypill for secondary prevention was devised. VB, AG and FG-A are/were employees of Ferrer Internacional and have been involved in the study design. All authors have been involved in the review of the systematic literature review, the model and the preparation and review of the manuscript.

Provenance and peer review Not commissioned; externally peer reviewed.

Data sharing statement No additional data are available.

Open Access This is an Open Access article distributed in accordance with the Creative Commons Attribution Non Commercial (CC BY-NC 4.0) license, which permits others to distribute, remix, adapt, build upon this work noncommercially, and license their derivative works on different terms, provided the original work is properly cited and the use is non-commercial. See: http:// creativecommons.org/licenses/by-nc/4.0/

\section{REFERENCES}

1. Dalton AR, Soljak M, Samarasundera E, et al. Prevalence of cardiovascular disease risk amongst the population eligible for the NHS Health Check Programme. Eur J Prev Cardiol 2013;20:142-50.

2. Neil HA, Perera R, Armitage JM, et al. Estimated 10-year cardiovascular risk in a British population: results of a national screening project. Int J Clin Pract 2008;62:1322-31.

3. Wang YC, McPherson K, Marsh T, et al. Health and economic burden of the projected obesity trends in the USA and the UK. Lancet 2011;378:815-25.

4. National Institute for Health and Care Excellence (NICE). MI-secondary prevention: secondary prevention in primary and secondary care for patients following a myocardial infarction [CG172]. London, UK: National Institute for Health and Care Excellence, 2013. http://guidance.nice.org.uk/CG172 (accessed Jun 2014).

5. Lafeber M, Spiering W, van der Graaf $Y$, et al. The combined use of aspirin, a statin, and blood pressure-lowering agents (polypill components) and the risk of vascular morbidity and mortality in patients with coronary artery disease. Am Heart J 2013;166:282-89.e1.

6. DeWilde S, Carey IM, Richards N, et al. Trends in secondary prevention of ischaemic heart disease in the UK 1994 2005: use of individual and combination treatment. Heart 2008;94:83-8.

7. Ramsay SE, Whincup PH, Wannamethee SG, et al. Missed opportunities for secondary prevention of cerebrovascular disease in elderly British men from 1999 to 2005: a population-based study. $J$ Public Health (Oxf) 2007;29:251-7.

8. Kotseva $\mathrm{K}$, Jennings CS, Turner EL, et al. ASPIRE-2-PREVENT: a survey of lifestyle, risk factor management and cardioprotective 
medication in patients with coronary heart disease and people at high risk of developing cardiovascular disease in the UK. Heart 2012;98:865-71.

9. Sheppard JP, Fletcher K, McManus RJ, et al. Maybe it's time for something a little different? The extent to which current pharmacotherapy practice conforms to national guidelines for stroke and cardiovascular disease prevention. Int J Stroke 2013;8:7.

10. Newby LK, LaPointe NM, Chen AY, et al. Long-term adherence to evidence-based secondary prevention therapies in coronary artery disease. Circulation 2006;113:203-12.

11. Wei L, Fahey T, MacDonald TM. Adherence to statin or aspirin or both in patients with established cardiovascular disease: exploring healthy behaviour vs. drug effects and 10-year follow-up of outcome. Br J Clin Pharmacol 2008;66:110-16.

12. Cascini S, Kirchmayer U, Bauleo L, et al. Evidence based drug therapy and medium-long-term outcomes in very old patients after acute myocardial infarction. Pharmacoepidemiol Drug Saf 2012;21:235.

13. Cotter G, Shemesh E, Zehavi M, et al. Lack of aspirin effect: aspirin resistance or resistance to taking aspirin? Am Heart J 2004;147:293-300.

14. Garcia Rodriguez LA, Cea Soriano L, Hill C, et al. Increased risk of stroke after discontinuation of acetylsalicylic acid: a UK primary care study. Neurology 2011;76:740-6.

15. Rajagopalan S, Vieira JL, Alemao E, et al. The impact of lipid-lowering treatment patterns on LDL-C reduction and goal attainment in secondary prevention in Germany. Prev Control 2006;2:15-26.

16. Jin J, Sklar GE, Min Sen Oh V, et al. Factors affecting therapeutic compliance: a review from the patient's perspective. Ther Clin Risk Manag 2008;4:269-86.

17. Perk J, De Backer G, Gohlke H, et al. European Guidelines on cardiovascular disease prevention in clinical practice (version 2012). The Fifth Joint Task Force of the European Society of Cardiology and Other Societies on Cardiovascular Disease Prevention in Clinical Practice (constituted by representatives of nine societies and by invited experts). Eur Heart $J$ 2012;33:1635-701.

18. Lafeber M, Spiering $\mathrm{W}$, Singh $\mathrm{K}$, et al. The cardiovascular polypill in high-risk patients. Eur J Prev Cardiol 2012;19:1234-42.

19. Muntner P, Mann D, Wildman RP, et al. Projected impact of polypill use among US adults: medication use, cardiovascular risk reduction, and side effects. Am Heart J 2011;161:719-25.

20. Sanz G, Fuster V. Fixed-dose combination therapy and secondary cardiovascular prevention: rationale, selection of drugs and target population. Nat Clin Pract Cardiovasc Med 2009;6:101-10.

21. Wald NJ, Law MR. A strategy to reduce cardiovascular disease by more than $80 \%$. BMJ 2003;326:1419-23.

22. Zeymer U, Junger $\mathrm{C}$, Zahn $\mathrm{R}$, et al. Effects of a secondary prevention combination therapy with an aspirin, an ACE inhibitor and a statin on 1-year mortality of patients with acute myocardial infarction treated with a beta-blocker. Support for a polypill approach Curr Med Res Opin 2011;27:1563-70.

23. National Institute for Health and Care Excellence (NICE). Guide to the methods of technology appraisal [PMG9]. London, UK: National Institute for Health and Care Excellence, 2013. http://publications. nice.org.uk/guide-to-the-methods-of-technology-appraisal-2013pmg9 (accessed 14 Jul 2014).

23a. Personal Social Services Research Unit (PSSRU). Unit costs of health \& social care 2013. 2014. http://www.pssru.ac.uk/projectpages/unit-costs/2013/ (accessed 14 Jul 2014).

24. Thom S, Poulter N, Field J, et al. Effects of a fixed-dose combination strategy on adherence and risk factors in patients with or at high risk of CVD: the UMPIRE randomized clinical trial. JAMA 2013;310:918-29.

25. Patel A, Cass A, Peiris D, et al. A pragmatic randomized trial of a polypill-based strategy to improve use of indicated preventive treatments in people at high cardiovascular disease risk. Eur J Prev Cardiol 2014. Published Online First 27 Mar 2014. doi: 10.1177/ 2047487314530382

26. Husereau D, Drummond M, Petrou S, et al. Consolidated Health Economic Evaluation Reporting Standards (CHEERS) - explanation and elaboration: a report of the ISPOR Health Economic Evaluation Publication Guidelines Good Reporting Practices Task Force. Value Health 2013;16:231-50.

27. de Vries ST, Keers JC, Visser R, et al. Medication beliefs, treatment complexity, and non-adherence to different drug classes in patients with type 2 diabetes. J Psychosom Res 2014;76:134-8.

28. Ross A, Jami H, Young HA, et al. Sync and swim: the impact of medication consolidation on adherence in Medicaid patients. J Prim Care Community Health 2013;4:240-4.
29. Gaziano TA, Opie LH, Weinstein MC. Cardiovascular disease prevention with a multidrug regimen in the developing world: a cost-effectiveness analysis. Lancet 2006;368:679-86.

30. Health and Social Care Information Centre. Prescription cost analysis England 2013. Leeds, UK: Health and Social Care Information Centre, 2014. http://www.hscic.gov.uk/catalogue/ PUB13887/pres-cost-anal-eng-2013-rep.pdf (accessed 14 Jul 2014).

31. Kotseva K, Wood D, De Backer G, et al. EUROASPIRE III: a survey on the lifestyle, risk factors and use of cardioprotective drug therapies in coronary patients from 22 European countries. Eur $J$ Cardiovasc Prev Rehabil 2009;16:121-37.

32. Ara R, Pandor A, Stevens J, et al. Prescribing high-dose lipid-lowering therapy early to avoid subsequent cardiovascular events: is this a costeffective strategy? Eur J Prev Cardiol 2012;19:474-83.

33. De Smedt D, Kotseva K, De Bacquer D, et al. Cost-effectiveness of optimizing prevention in patients with coronary heart disease: the EUROASPIRE III health economics project. Eur Heart $J$ 2012;33:2865-72.

34. Heeg B, Damen J, Van Hout B. Oral antiplatelet therapy in secondary prevention of cardiovascular events: an assessment from the payer's perspective. Pharmacoeconomics 2007;25:1063-82.

35. Scuffham PA, Chaplin S. An economic evaluation of fluvastatin used for the prevention of cardiac events following successful first percutaneous coronary intervention in the UK. Pharmacoeconomics 2004;22:525-35.

36. Scuffham PA, Chaplin S. A cost-effectiveness analysis of fluvastatin in patients with diabetes after successful percutaneous coronary intervention. Clin Ther 2005;27:1467-77.

37. Selak V, Elley CR, Bullen C, et al. Effect of fixed dose combination treatment on adherence and risk factor control among patients at high risk of cardiovascular disease: randomised controlled trial in primary care. BMJ 2014;348:g3318.

38. British Heart Foundation. Cardiovascular disease statistics 2014. London, UK: British Heart Foundation, 2014. http://www.bhf.org.uk/ research/heart-statistics/morbidity/prevalence.asp (accessed $14 \mathrm{Jul}$ 2014).

39. Smolina K, Wright FL, Rayner M, et al. Incidence and 30-day case fatality for acute myocardial infarction in England in 2010: national-linked database study. Eur J Public Health 2012;22:848-53.

40. Office for National Statistics (ONS). Population projections. 2014 http://www.ons.gov.uk/ons/taxonomy/index.html?nscl=Population +Projections (accessed Jun 2014)

41. Department of Health (UK). National schedule of reference costs: spell costs. 2013. https://www.gov.uk/government/publications/nhsreference-costs-2012-to-2013 (accessed 14 Jul 2014).

42. Saka RO, McGuire A, Wolfe CDA. Economic burden of stroke in England. London, UK: University of London, 2005. http://www.nao. org.uk/wp-content/uploads/2005/11/0506452_economic_analysis.pdf (accessed 12 Mar 2015).

43. Taylor DC, Pandya A, Thompson D, et al. Cost-effectiveness of intensive atorvastatin therapy in secondary cardiovascular prevention in the United Kingdom, Spain, and Germany, based on the Treating to New Targets study. Eur J Health Econ 2009;10:255-65.

44. Ward S, Lloyd Jones M, Pandor A, et al. A systematic review and economic evaluation of statins for the prevention of coronary events. Health Technol Assess 2007;11:1-160, iii-iv.

45. Choudhry NK, Patrick AR, Antman EM, et al. Cost-effectiveness of providing full drug coverage to increase medication adherence in post-myocardial infarction Medicare beneficiaries. Circulation 2008;117:1261-8

46. Saha SA, Molnar J, Arora RR. Tissue ACE inhibitors for secondary prevention of cardiovascular disease in patients with preserved left ventricular function: a pooled meta-analysis of randomized placebo-controlled trials. J Cardiovasc Pharmacol Ther 2007;12:192-204.

47. Baigent C, Blackwell L, Collins R, Antithrombotic Trialists (ATT) Collaboration. Aspirin in the primary and secondary prevention of vascular disease: collaborative meta-analysis of individual participant data from randomised trials. Lancet 2009;373:1849-60.

48. Yusuf S, Sleight P, Dagenais G. The HOPE (Heart Outcomes Prevention Evaluation) Study. FDA briefing: a large, randomized trial of the ACE inhibitor, ramipril, and vitamin $E$ in patients at high risk of cardiovascular events. Silver Spring, MD: U.S. Food and Drug Administration, 2000. http://www.fda.gov/ohrms/dockets/ac/00/ backgrd/3612b1d.pdf (accessed 14 Jul 2014).

49. Vale N, Nordmann AJ, Schwartz GG, et al. Statins for acute coronary syndrome. Cochrane Database Syst Rev 2011;(6):CD006870.

50. British National Formulary. 2014. http://www.bnf.org/bnf/index.htm (accessed 14 Jul 2014). 